\title{
Norcantharidin triggers apoptotic cell death in non-small cell lung cancer via a mitophagy-mediated autophagy pathway
}

\author{
Zhilong Liu ${ }^{1 \#}$, Baoxia $\mathrm{Li}^{2 \#}$, Mingrong $\mathrm{Cao}^{1}$, Jianwei Jiang ${ }^{3}$ \\ ${ }^{1}$ Department of General Surgery, The First Affiliated Hospital, Jinan University, Guangzhou, China; ${ }^{2}$ State Laboratory of Oncology in South China, \\ Sun Yat-sen University Cancer Center, Guangzhou, China; ${ }^{3}$ Department of Biochemistry, Medical College, Jinan University, Guangzhou, China \\ Contributions: (I) Conception and design: Z Liu, B Li, J Jiang; (II) Administrative support: Z Liu, B Li; (III) Provision of study materials or patients: \\ Z Liu, M Cao, J Jiang; (IV) Collection and assembly of data: Z Liu, B Li, M Cao; (V) Data analysis and interpretation: All authors; (VI) Manuscript \\ writing: All authors; (VII) Final approval of manuscript: All authors. \\ \#These authors contributed equally to this work. \\ Correspondence to: Jianwei Jiang. Department of Biochemistry, Medical College, Jinan University, Guangzhou 510632, China. Email: jjw703@163.com.
}

\begin{abstract}
Background: Norcantharidin (NCTD) is known to impact on cell progression in many cancers; however, its activity in non-small cell lung cancer (NSCLC) has not yet been characterized. In the present study, we set out to determine the cytotoxic effects of NCTD on the proliferation and apoptosis on A549 cells and their underlying mechanisms.

Methods: NSCLC cell line A549 cells were cultured. A549 cells were treated with different concentrations of NCTD. Cell proliferation was detected by MTT and cell clone formation assay. Cell cycle and apoptosis were detected by flow cytometry. After A549 cells were treated with NCTD for 24 hours, the mitochondrial membrane potential was measured. The protein expression of Bcl-2, Bax, light chain 3 (LC3), et al. was tested by western blot. The expression of LC3 and Tom 20 protein was detected by immunofluorescence.

Results: NCTD suppressed the proliferation of NSCLC cells while decreasing mitochondrial membrane potential and inducing G2/M phase arrest. NCTD induced apoptosis, as demonstrated by increased B-cell lymphoma 2/Bcl-2-associated $\mathrm{X}$ protein and Bcl-2-associated X protein/myeloid cell leukemia 1 ratios. Aside from autophagy, NCTD induced mitophagy, with an increase in LC3 expression and a decrease in sequestosome 1 (p62) expression in the cytoplasm, accompanied by increased levels of Phospho-adenosine 5'-monophosphate -activated protein kinase (p-AMPK), Phospho-c-Jun NH2-Terminal Kinase (p-JNK), and Phospho-c-jun (p-c-jun) and a decreased level of Phospho-protein kinase B (p-AKT).
\end{abstract}

Conclusions: This study has elucidated that NCTD restrains NSCLC cell progression via regulation of AMPK/mammalian target of rapamycin (mTOR)/uncoordinated 51-like kinase 1 (ULK1)/JNK pathways. This evidence provides insight into a novel treatment for NSCLC.

Keywords: Norcantharidin (NCTD); autophagy; apoptosis; non-small cell lung cancer (NSCLC)

Submitted Mar 31, 2021. Accepted for publication Jun 02, 2021.

doi: $10.21037 / \mathrm{atm}-21-2360$

View this article at: http://dx.doi.org/10.21037/atm-21-2360

\section{Introduction}

Lung cancer causes more fatalities than any other malignancy (1), and non-small cell lung cancer (NSCLC) accounts for $75-80 \%$ of all lung cancer cases (2). Despite significant advances in its diagnosis and treatment, lung cancer still has a poor prognosis, with a 5 -year overall survival rate of only $15 \%$ (3). Chemotherapy is the most frequently used treatment for lung cancer. However, with side effects of chemotherapy including myelosuppression, neutropenia, and thrombocytopenia, an actionable approach or novel drugs to treat this disease must be developed.

Norcantharidin (NCTD) is a normethyl compound of cantharidin (CTD) found in Mylabris. Pharmacological 
studies have evidenced the effects of CTD on the lung and intestine (4). NCTD exhibits selective toxicity toward cancer cells, with similar efficacy as CTD but with fewer side effects; thus, it is widely applied in clinical practice (5). The ability of NCTD to enhance cancer cell apoptosis has been reported. However, the relationship between NSCLC and NCTD has not been thoroughly elucidated, and although NCTD is involved in autophagy, the related molecular mechanism is poorly understood.

Autophagic regulation is expected to become an alternative treatment for malignant tumors. Mitophagy triggers mitochondrial depolarization (6). The mitophagic process is initiated to maintain cell survival, but mitophagy can also lead to cell death if cell injury is prolonged (7). In the present study, we found that NCTD caused a decline in mitochondrial membrane potential $(\Delta \Psi \mathrm{m})$, activated Adenosine 5'-monophosphate-activated protein kinase (AMPK) and inhibited the phosphatidylinositol 3-kinase (PI3K)/protein kinase B (Akt)/mammalian target of rapamycin (mTOR) signaling pathway, induced A549 cell mitophagy and autophagy, and subsequently caused mitochondrial-dependent apoptosis.

We present the following article in accordance with the MDAR reporting checklist (available at http://dx.doi. org/10.21037/atm-21-2360).

\section{Methods}

\section{Cell culture and reagents}

NSCLC cell line A549 (Shanghai Institute of Cell Biology, China) was cultured in Roswell Park Memorial Institute (RPMI) Medium 1640 containing $10 \%$ fetal bovine serum (FBS, Gibco Corporation, Carlsbad, CA, USA), $1 \mathrm{mM}$ glutamine, $1.5 \mathrm{~g} / \mathrm{L}$ sodium bicarbonate, and $1 \%$ penicillin/ streptomycin. Primary antibodies included anti-B-cell lymphoma-2 (Bcl-2), anti-BCL2-Associated X (Bax), antipoly ADP ribose polymerase (PARP)-1, anti-myeloid cell leukemia 1 (Mcl-1), anti-Bcl-xL, anti-caspase-3, anticaspase-8, anti-caspase-9 anti-PARP, anti-AMPK, antip-AMPK, anti-Akt, anti-p-Akt, anti-c-Jun, Phospho-cjun (p-c-Jun), anti-c-Jun NH2-Terminal Kinase (JNK), anti-uncoordinated 51-like kinase 1 (ULK1), anti-p-JNK, anti-mTOR, anti-Beclin-1, anti-light chain 3 (LC3), antisequestosome 1 (p62) and anti-GAPDH, anti-DAPI, and anti-Tom20 were purchased from Cell Signaling Technology Inc (Beverly, MA, USA). Anti-cyclin A, anticyclin B2, anti-cyclin D1, anti-cyclin D3, anti-p21, and
anti-Cdc2 were purchased from Sigma-Aldrich (Munich, Germany), as was analytical-grade NCTD.

\section{MTT assay}

Cell proliferation was assessed using the MTT assay (8). Briefly, cells were seeded in 96 -well plates $\left(5 \times 10^{3}\right.$ cells $\left./ \mathrm{mL}\right)$, and 24 hours later, they were cultured with NCTD (0, $0.16075,0.3125,0.625,1.25,2.5,5,10,20,40$, and $80 \mu \mathrm{M})$. Untreated cells served as a control group. After 72-hour culture, MTT solution $(10.0 \mu \mathrm{L} /$ well $)$ was added to the cells, and they were cultured for a further 4-6 hours. After dissolving purple formazan crystals in $200 \mu \mathrm{L}$ dimethyl sulfoxide (DMSO), we used a spectrophotometer (Thermo Molecular De-vices Co., Union City, USA) to analyze the absorbance at $570 \mathrm{~nm}$. The inhibition rate was calculated as (A570 control cells $-\mathrm{A} 570_{\text {treated cells }}$ ) $/ \mathrm{A} 570_{\text {control cells }} \times 100 \%$, and the $\mathrm{IC}_{50}$ was determined using the logit method.

\section{Clone formation assay}

A clone formation assay was performed by plating cells in 12-well plates (300 cells/well) in 100\% RPMI Medium 1640 for 24 hours and adding NCTD to the microplates. Following culture for 7 days, the petri dish was washed with PBS twice, $4 \%$ paraformaldehyde added to fix the cells. After 15 minutes, paraformaldehyde removed, the cell was dyed in $0.1 \%$ crystal violet (Solarbio, Beijing, China). After 10-30 minutes, the staining solution was rinsed off with running water. After drying, the number of cell colonies was observed and recorded under the microscope.

\section{Flow cytometry}

Cells were plated and treated with NCTD $\left(2 \times 10^{5}\right.$ cells/well $)$. The cells were then stained with Annexin V-FITC/PI (Becton Dickinson, USA). Apoptotic cells were detected with a flow cytometer (Beckman Coulter, Fullerton, California, USA) and CellQuest software (FCM, Becton Dickinson, USA).

\section{Cell cycle analysis}

Briefly, A549 cells were incubated with NCTD for 24 hours, fixed in $70 \%$ ethanol overnight, and incubated with 20 units/mL RNase I and $50 \mu \mathrm{g} / \mathrm{mL}$ propidium iodide (Becton Dickinson, USA) for 30 minutes. Flow cytometry was carried out to determine the cellular DNA content. 
The results were analyzed with CellQuest software (FCM, Becton Dickinson, USA).

\section{$\mathcal{F C}-1$ dye}

Cells were plated and treated with NCTD $\left(2 \times 10^{5}\right.$ cells/well $)$ for 24 hours. Then, the cells were re-suspended and added with JC-1 dye (Beyotime Co, Hangzhou, China) on slides. The slides were viewed under a fluorescence microscope, and the $\Delta \Psi \mathrm{m}$ was measured (from red to green).

\section{Western blot}

A549 cells were treated with NCTD for 24 hours and then lysed with $5 \times$ sodium dodecyl sulfate (SDS) sample buffer. Proteins were separated and transferred to polyvinylidene difluoride (PVDF) membranes. After being blocked with $5 \%$ nonfat dry milk/tris-buffered saline with Tween 20 , the membranes were probed with antibodies against Bcl2, Bax, PARP-1, Mcl-1, Bcl-xL, Bcl-2, Bax, caspas-3/8/9, PARP, AMPK, p-AMPK, Akt, p-Akt, c-Jun, p-c-Jun, JNK, ULK1, p-JNK, mTOR, Beclin-1, LC3, p62, cyclin A, cyclin B2, cyclin D1, cyclin D3, p21, Cdc2, and GAPDH (1:1,000 dilution in blocking buffer) at $4{ }^{\circ} \mathrm{C}$ overnight. They were then probed with secondary antibodies for 1 hour and finally visualized using SuperSignal West Pico reagents (NCI5079, Thermo).

\section{Immunofluorescence}

As previously described (9), cells were plated, treated with $0 \mu \mathrm{M}$ NCTD (the control group) or $5 \mu \mathrm{M}$ NCTD for 24 hours, and then fixed with $4 \%$ paraformaldehyde for 10 minutes. The cells were permeabilized with $0.25 \%$ Triton-X 100 and incubated in blocking solution. Following that, the cells were incubated with LC3 antibody ( $1: 200$ dilution in blocking buffer) or Tom 20 antibody (1:200 dilution in blocking buffer) for 24 hours, and then with fluorescein isothiocyanate (FITC)-conjugated secondary antibody for 2 hours at room temperature. After staining with DAPI $(1 \mathrm{mg} / \mathrm{mL})$ for 5 minutes, the cells were examined under a confocal laser microscope.

\section{Statistical analysis}

Data were processed with SPSS version 16.0 (Inc., Chicago, IL, USA) and presented as the mean \pm standard deviation (SD). All assays were performed at least 3 times.

\section{Results}

\section{Inbibitive effects of NCTD on NSCLC cell growth}

The results of NCTD inhibiting NSCLC cell viability were displayed in Figure 1A,B,C). The molecular structure of CTD and NCTD was shown in Figure $1 A$. To determine the effect of NCTD on cell growth, the cell viability of the NSCLC cell line A549 and the human hepatic cell line LO2 was detected following treatment with NCTD (Figure 1B). NCTD exhibited cytotoxicity in A549 cells and moderate cytotoxic activity in LO2 cells. NCTD strongly inhibited the growth of cancer cells, and decreased cell viability in a dose-dependent manner; the $\mathrm{IC}_{50}$ value was $13.1 \mu \mathrm{M}$. Additionally, A549 cell proliferation was strongly suppressed in the presence of NCTD (Figure 1C).

\section{Impact of NCTD on the cell cycle distribution of A549 cells}

Because cell growth inhibition is typically associated with cell cycle arrest, we used propidium iodide staining to examine the cell cycle distribution of NCTD-treated A549 cells. The results of NCTD inducing cell cycle arrest in A549 cells were shown in Figure $2 A, B, C$. We observed that NCTD treatment caused G2/M phase arrest (Figure $2 A$ ). To further investigate the effect of NCTD on the cell cycle, we examined the expression of cell cycle-associated proteins through western blot analysis. The expression of p21 was also found to be increased, while that of cdc2 was decreased (Figure 2B). NCTD treatment induced decreases in the expression levels of cyclin D3, cyclin E2, and the G1 phase- and S phase-associated proteins, and increases in the expression levels of cyclin B1, cyclin A, and the G2/ $M$ phase-associated proteins (Figure 2C). G2 to $M$ phase transition depended on the activity of the cdc2/cyclin B complex (M phase-promoting factor). In turn, p21 reduced cdc2/cyclin B complex activity and increased the expression of cyclin A and cyclin B1. Collectively, these results suggest that NCTD might cause cell cycle arrest by upregulating the expression of $\mathrm{p} 21$.

\section{NCTD induced apoptosis and reduced the $\Delta \Psi_{m}$}

The results of NCTD inducing apoptosis and reducing mitochondrial membrane potential were shown in Figure $3 A, B, C$. We used flow cytometry to explore the function of NCTD in cell apoptosis (Figure $3 A, C$ ). After treatment with NCTD, the proportion of apoptotic cells increased from $4.0 \%$ to $40.1 \%$, suggesting that NCTD 


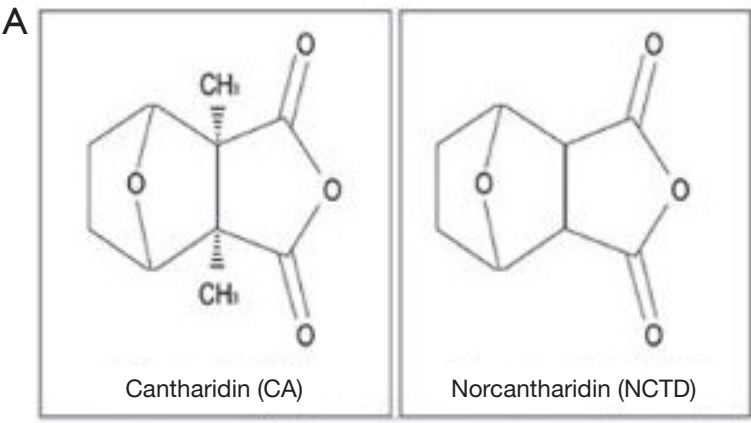

Structures of cantharidin (CA) and norcantharidin (NCTD)

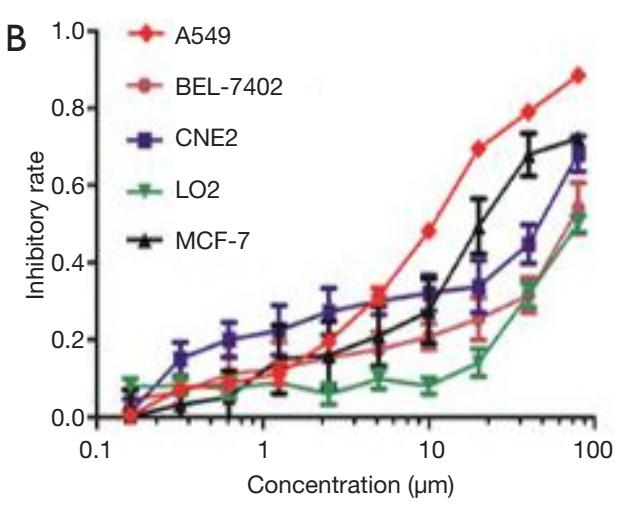

C

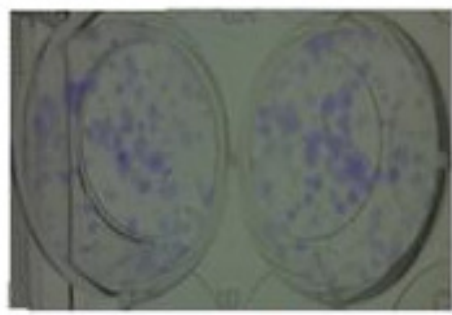

$0 \mu \mathrm{m}$

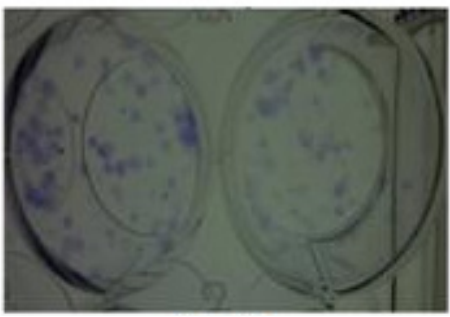

$1.5 \mu \mathrm{m}$

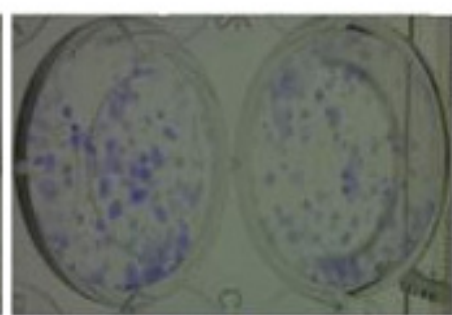

$0.325 \mu \mathrm{m}$

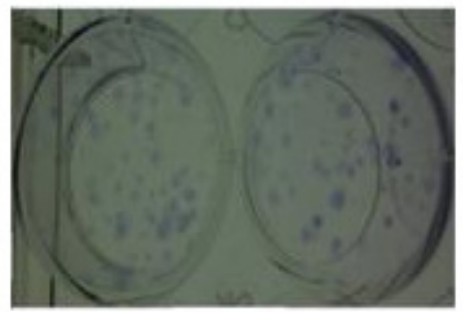

$3 \mu \mathrm{m}$

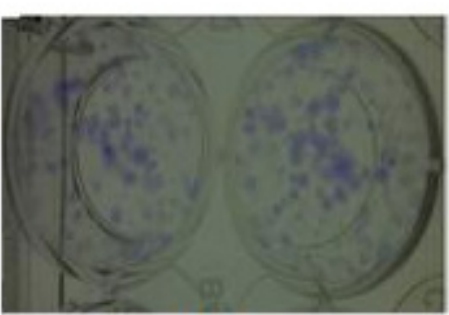

$0.75 \mu \mathrm{m}$

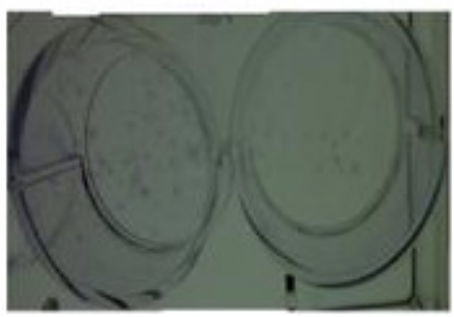

$6 \mu \mathrm{m}$

Figure 1 NCTD inhibits NSCLC cell viability. (A) The molecular structure of CTD and NCTD. (B) Cell viability after treatment with NCTD, as measured by MTT assay. (C) Results of colony-forming assay performed after treatment with NCTD, images were magnified at 20×. NCTD, norcantharidin; NSCLC, non-small cell lung cancer.

induced apoptosis of A549 cells in a dose-dependent manner.

Loss of $\Delta \Psi_{m}$ directly determines cell death. Therefore, we used flow cytometry to explore the impact of NCTD on mitochondria. As shown in Figure 3B, treatment with NCTD led to a decline in $\Delta \Psi m$.

\section{NCTD modulates the expression of Bcl-2 family proteins and caspase-3/9 in A549 cells}

Next, we examined the expressions of Bcl-2 and caspase families in NCTD-treated A549 cells (Figure 4A,B). As shown in Figure 4A, after 24 hours of NCTD treatment, the levels of Bcl-2 and Mcl-1 were markedly downregulated. However, the expressions of 2 other pro-apoptotic proteins,
Bax and Bcl-xl, were not obviously altered. NCTD activated caspase-3/9 and PARP (Figure 4B), but pro-caspase- 8 was not significantly altered. These results suggest that NCTD induced A549 cell apoptosis via the mitochondrial signaling pathway.

\section{NCTD-induced autophagy and mitophagy}

To investigate NCTD-induced autophagy in A549 cells, we examined the expression levels of LC3 and p62. The results of NCTD enhancing autophagy and mitophagy in A549 cells were shown in Figure 5A,B,C,D. Treatment of A549 cells with NCTD accelerated the conversion of LC3-I to LC3-II, while reducing the expression of p62 (Figure 5A). The protein expression of TOM20 was shown 


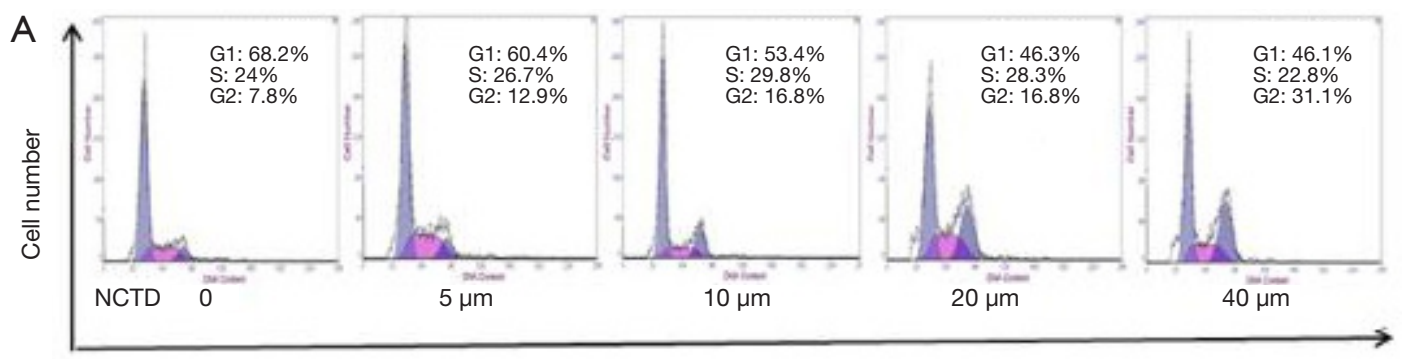

DNA content
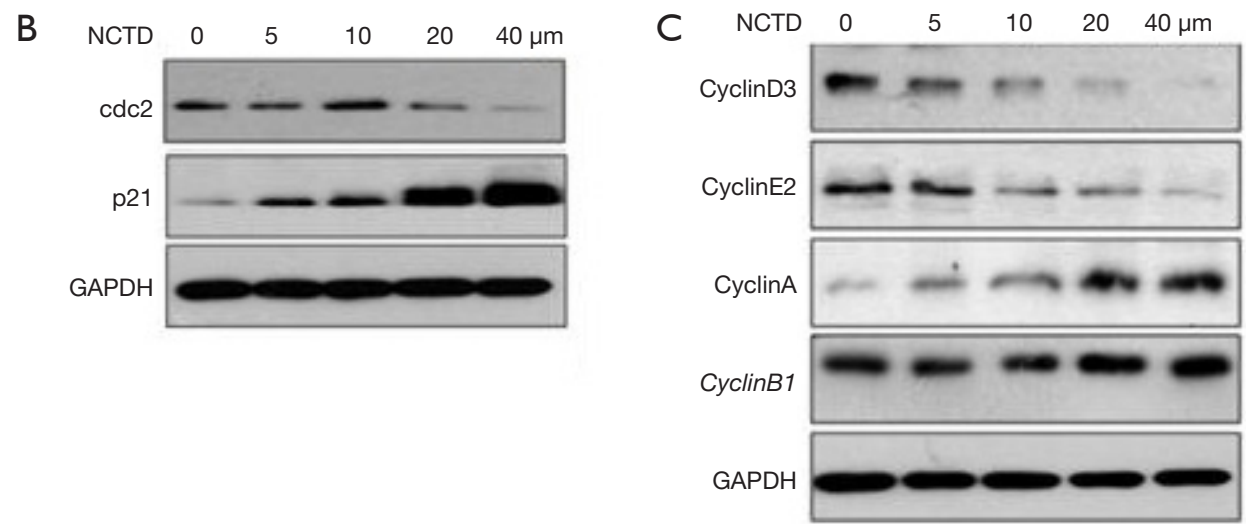

Figure 2 NCTD induced cell cycle arrest in A549 cells. (A) Results of flow cytometry of cell cycle after treatment with NCTD (0, 5, 10, 20, and $40 \mu \mathrm{M})$. (B) Western blot analysis of cell cycle-associated proteins after treatment with NCTD. (C) Western blot analysis of cyclin family members. NCTD, norcantharidin.

in Figure 5B. LC3 is a component of the autophagosomal membranes, and punctate LC3 accumulation was observed in the cytoplasm (Figure 5C), which confirmed that NCTD enhanced autophagy in A549 cells. The depolarization of mitochondria usually takes place during mitophagy (6). We found that NCTD treatment decreased the $\triangle \Psi \mathrm{m}$. We next identified the presence of mitophagy after treatment to further study the impact of NCTD on autophagy. TOM20 is a mitochondrial outer membrane protein, the decreased expression of which indicates mitophagy (10). NCTD reduced the protein expression of TOM20, with mitochondrial clustering around the nucleus (Figure 5D), which indicated that mitophagy occurred.

\section{The effects of NCTD on the expression of Akt/mTOR patbway-and the $\mathcal{F N K / c - j u n ~ p a t b w a y - r e l a t e d ~ f a c t o r s / ~}$ proteins}

The results of NCTD affecting intracellular signaling pathways in A549 cells were shown in Figure 6A,B. The PI3K/Akt/mTOR, AMPK/mTOR, and JNK pathways are involved in a variety of physiological functions, including cell metabolism, proliferation, survival, protein synthesis. We therefore analyzed the expression of mTOR, p-Akt, and p-AMPK in A549 cells by western blotting. The expression levels of p-Akt and mTOR were down-regulated with increasing concentrations of NCTD (Figure 6A). In contrast, the expression levels of p-AMPK and ULK1 were increased. Moreover, p-JNK and its downstream protein p-c-jun were upregulated (Figure 6B). These results indicate that the activation of the Akt/mTOR pathway and the JNK/c-jun pathway, and the inhibition of AMPK/mTOR signaling were involved in NCTD-induced apoptosis and autophagy in A549 cells.

\section{Discussion}

NCTD is known to suppress cancer cell progression, but its effect on NSCLC cells has remained elusive. The present research has uncovered a mechanism via which NCTD inhibits the growth of A549 cells. We found that NCTD suppressed cell growth, as demonstrated by a reduced number of clones (Figure 1B,C). Abnormal cell proliferation is usually driven by cell cycle disruption and is common in a 

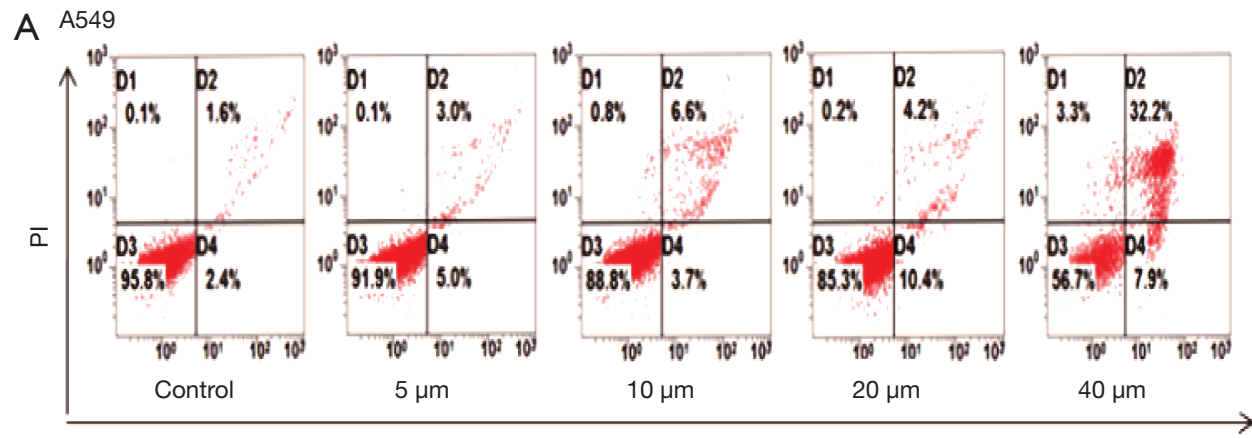

Annexin

B A549

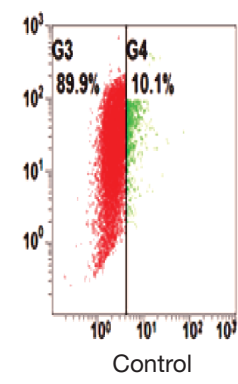

C
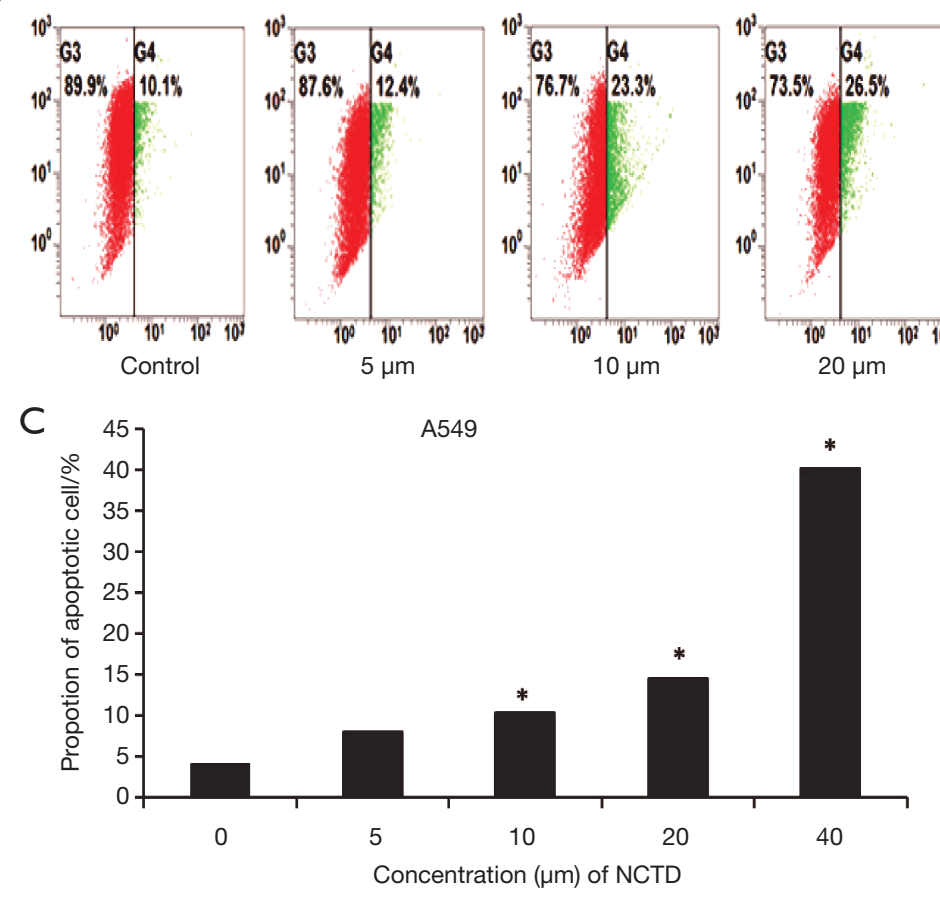
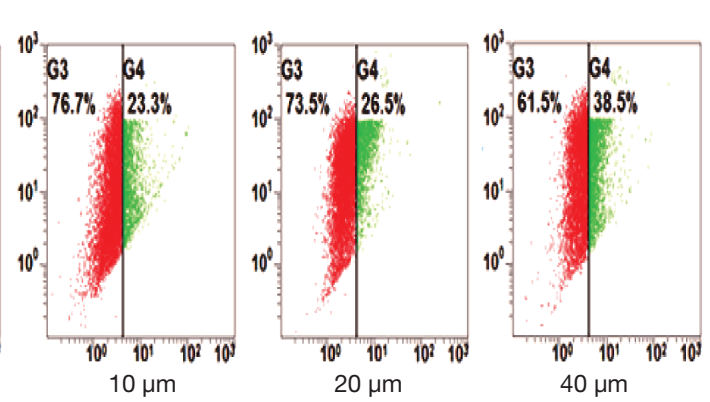

Figure 3 NCTD induces apoptosis and reduces mitochondrial membrane potential. (A) Flow cytometry image of apoptosis. (B) Quantification of mitochondrial membrane potential after the addition of $0,5,10,20$, or $40 \mu M$ of NCTD was evaluated by flow cytometry. (C) Quantification of apoptotic cells $(\mathrm{n}=3)$; ${ }^{*} \mathrm{P}<0.05$ vs. untreated group. NCTD, norcantharidin.

variety of cancers (11). Our current work demonstrates that NCTD-induced apoptosis is correlated with cell cycle arrest via the regulation of the expression of related genes, such as CDKs and CDKIs $(12,13)$. We found that cells accumulated in the G2 phase, and that NCTD downregulated cyclin D3 and cyclin E2 while upregulating cyclin A and cyclin B (Figure 2A,C). These results suggest that NCTD inhibits cell proliferation, probably by influencing cellular mitosis. It was indicated that $\mathrm{p} 21$ cooperated with CDK and cyclin compounds to affect the cell cycle. G2 to $M$ phase transition depends on the activity of the cdc2/cyclin B complex (M phase-promoting factor). Our results (Figure $2 B, C$ ) showed that p21 suppressed cdc2/cyclin B complex despite increases in the levels of cyclin A and B; therefore, we concluded that NCTD induced cell cycle arrest in the G2 phase through increasing the expression of $\mathrm{p} 21$. Akt might also inhibit $\mathrm{p} 21$ expression through the phosphorylation and activation of MDM2 and the downregulation of $\mathrm{p} 21$. Our results indicate that after NCTD treatment, p-AMPK expression increased, and the expression of p-Akt decreased (Figure $6 A$ ). In short, NCTD activates AMPK and suppresses cdc2/cyclin B complex, thereby blocking the cell cycle. 

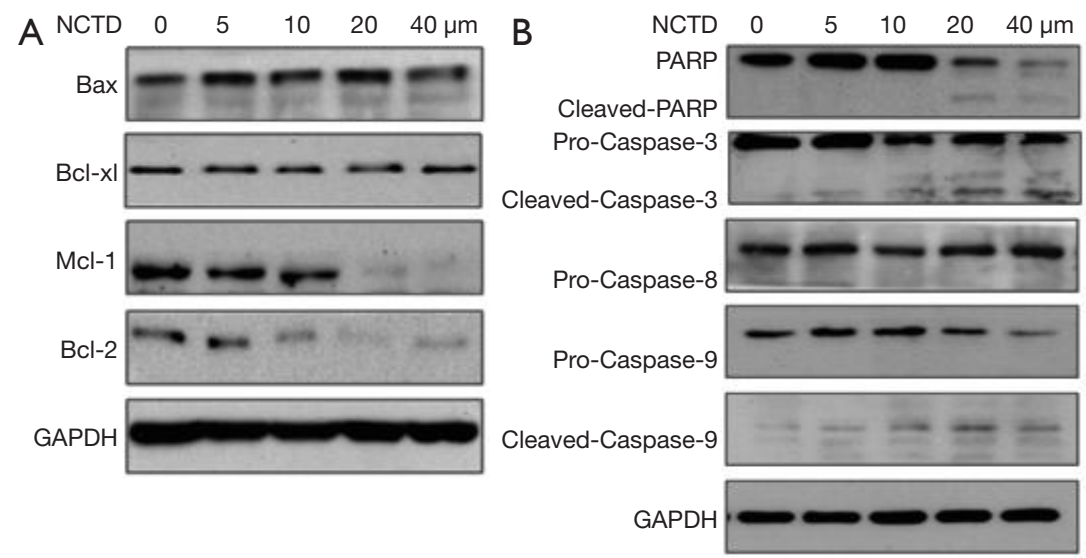

Figure 4 NCTD alters the expression of Bcl-2 and caspase-3/9/PARP in A549 cells. (A) Western blot analysis of Bcl-2 after NCTD treatment. (B) Western blot analysis of caspase- 3 and 9 and PARP. NCTD, norcantharidin.

A

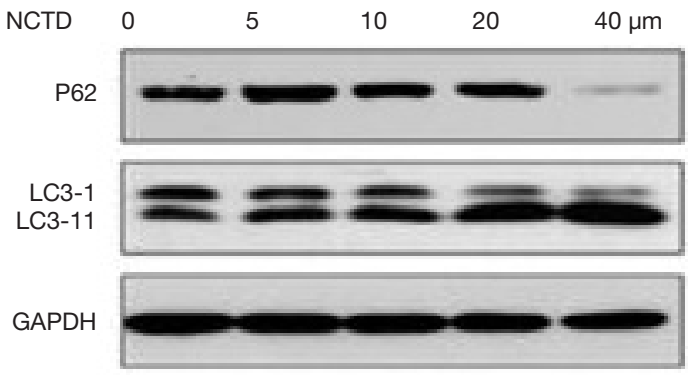

C

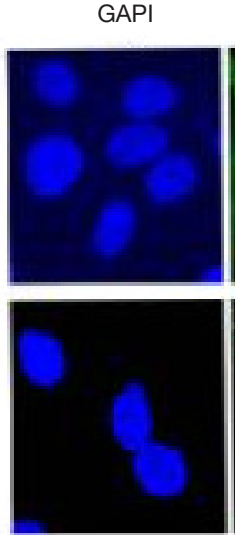

LC3

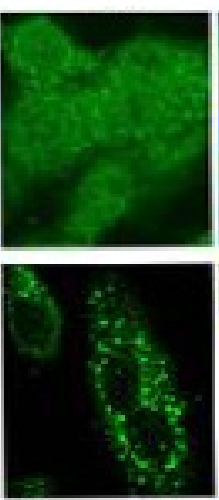

Merge

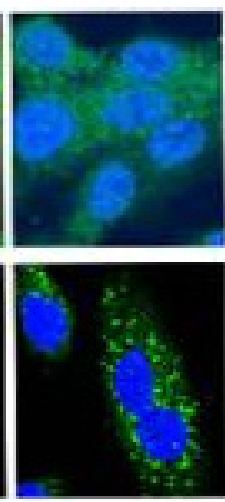

B

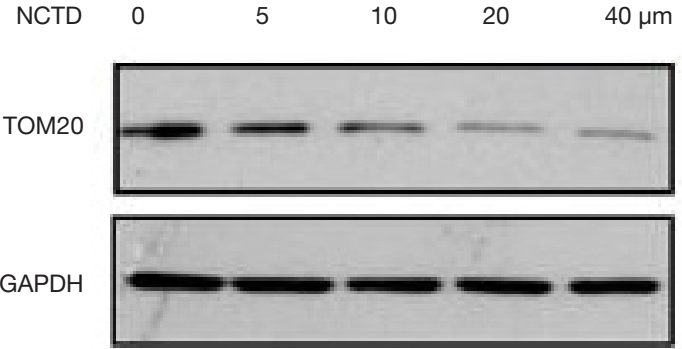

$\mathrm{D}$

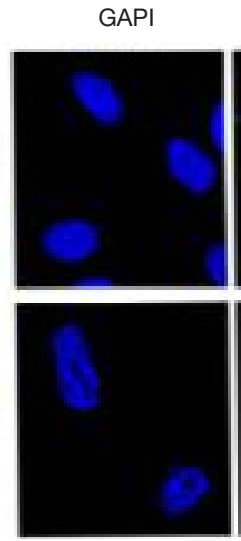

TOM20
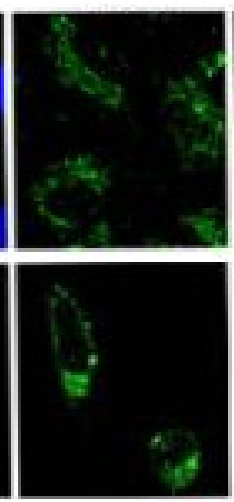

Merge

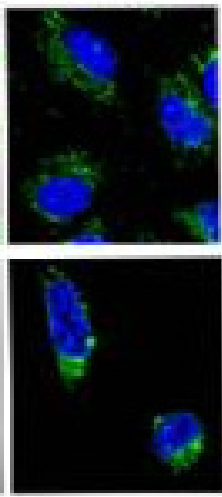

Figure 5 NCTD enhances autophagy and mitophagy in A549 cells. (A) Western blot analysis of LC3 and p62 expression after NCTD treatment. (B) Punctate formations of LC3 proteins in A549 cells after treatment with NCTD. (C) LC3 expression by immunofluorescence after NCTD treatment in A549 cells (200x). (D) TOM20 expression by immunofluorescence after NCTD treatment in A549 cells (200x). A549 cells were treated with different concentrations of NCTD $(0$ or $5 \mu M)$ for 24 hours, and the cells were then stained with anti-TOM20. Finally, the cells were examined using a fluorescence confocal microscope. Green: FITC-labeled LC3 or TOM20; Blue: DAPI-labeled nuclei. NCTD, norcantharidin; FITC, fluorescein isothiocyanate; DAPI, 4',6-diamidino-2-phenylindole. 

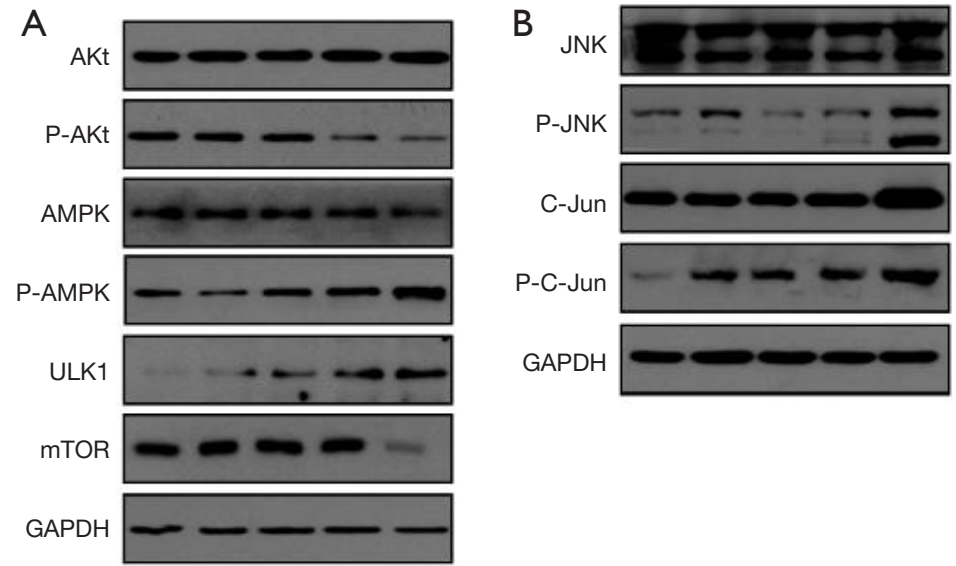

Figure 6 NCTD affects intracellular signaling pathways in A549 cells. (A) Western blot of p-AMPK, p-Akt, ULK1, and mTOR expression after NCTD treatment. (B) Western blot of JNK, p-JNK, c-jun, and p-c-jun expression after NCTD treatment. NCTD, norcantharidin.

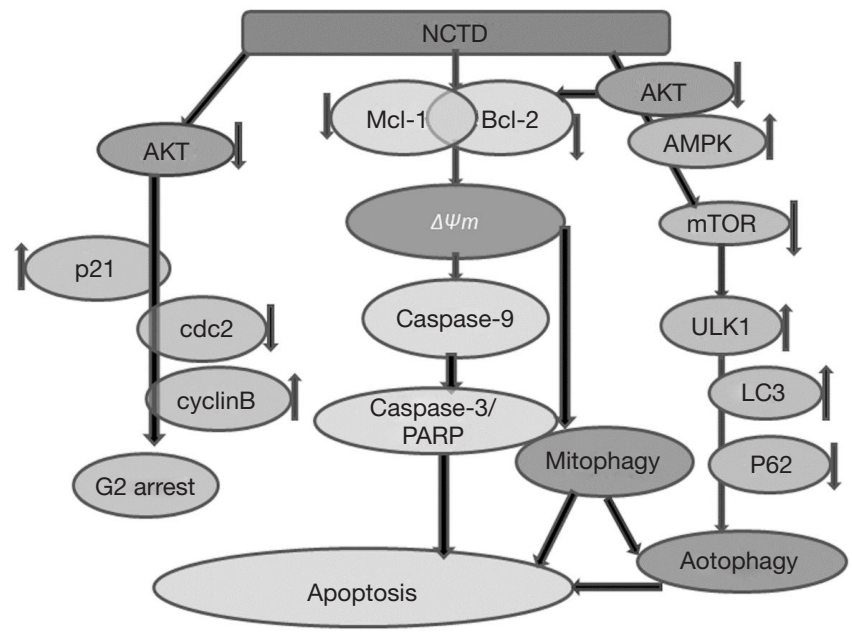

Figure 7 Schematic diagrams depicting NCTD-induced inhibition of the Akt/mTOR pathway and activation of the AMPK/mTOR/ ULK1 and JNK signaling pathways. NCTD, norcantharidin.

The significance of apoptosis in the treatment of cancer has frequently been reported. Here, we found that the administration of NCTD induced apoptosis (Figure 3A,C). Pro-apoptotic activity is linked to caspase- 3 activation, as well as PARP-1 cleavage (14). When Mitochondrial membrane permeability was augmented, the $\Delta \Psi_{m}$ was reduced, producing pro-apoptotic molecules such as cytochrome $\mathrm{C}$, and eventually activating caspase-3 $(15,16)$. Importantly, we found that NCTD treatment decreased $\Delta \Psi_{m}$ in A549 cells. Bcl-2 and caspase feature heavily in cell apoptosis. In our study, we found that the expression of Bcl-
2 and Mcl-1 decreased after NCTD treatment. However, the levels of Bax and Bcl-xL remained unchanged, with elevated Bax/Bcl-2 and Bax/Mcl-1 ratios (Figure $4 A$ ), which led to the activation of caspase-3/9 (Figure 4B). The activation of caspase- 3 cleaves PARP and induces apoptosis (9). As caspase- 8 did not show any obvious alterations, we speculate that NCTD-induced apoptosis rarely cooperates with the exogenous apoptosis pathway (Figure 4B).

Autophagy, a crucial process, directly affects cellular processes (17). Abnormal autophagy can consume intracellular components and lead to cell death (18). Autophagic regulation has been indicated as being instrumental to cancer treatment. In this study, NCTD treatment facilitated the transition of LC3-I to LC3-II, decreased p62 level (Figure 5A), and enhanced punctate LC3 accumulation (Figure 5C).

Mitophagy is a mitochondrial recycling process that primarily promotes cell survival; however, it can also cause cell death if prolonged cellular injury occurs. Persistent mitophagy leads to autophagy (6). Mitophagy has been highlighted as a typical symbol of autophagy and is triggered by mitochondrial damage (19). Abnormal TOM20 protein expression may be a sign of mitophagy (10). In the present study, we found that NCTD caused a decline in $\triangle \Psi_{m}$ in A549 cells. Treatment with NCTD reduced the protein expression of TOM20 (Figure 5B) and also induced characteristic clustering of the mitochondria around the nucleus (Figure 5D).

The Akt/mTOR pathway is crucial to cell progression, and its activation influences cancer progression $(20,21)$. Some antitumor drugs stimulate autophagic apoptosis 
of tumor cells via this pathway $(17,22)$. mTOR is a key regulatory factor in autophagy, with its activation inducing autophagic inhibition (23). The upstream mTOR signaling pathway regulates various physiological functions, including cell growth and the cell cycle, mainly through the PI3K/ Akt/mTOR pathway and the non-dependent PI3K/ Akt pathway. Akt is a kinase upstream of AMPK, which belongs to the serine/threonine protein kinase subfamily. Akt inhibits AMPK and activates mTOR via the AMPKTSC-MTOR pathway $(24,25)$. In mammalian cells, NCTD activates AMPK (26). AMPK induces autophagy via ULK1 protein or mTOR activation (27). Our work has demonstrated that NCTD decreased p-Akt and mTOR expression, while increasing the levels of p-AMPK and ULK1, suggesting that NCTD promotes autophagy in A549 cells (Figure 6A). C-JNK is activated by diverse stimuli, including cytokines and reactive oxygen species. It has involvement in multiple processes, including cell death and survival, and insulin signaling (28). JNK-mediated phosphorylation of Bcl-2 at Ser70, Bcl-xL at Thr47 and Thr115, and Mcl-1 at Ser121 and Thr163 can inactivate these anti-apoptotic proteins in response to cellular stresses. Meanwhile, JNK-mediated phosphorylation of Ser128, Bim at Ser65, BimL at Thr56, and Bax at Thr67 promotes these pro-apoptotic proteins (29). In this study, we found that NCTD activated JNK and the downstream protein c-jun to promote cell apoptosis and autophagy (Figure 6B).

As shown in Figure 7, NCTD treatment first activated Akt, then upregulated p21 and inhibited the activity of cdc2/cyclin B. Finally, it induced cell cycle arrest in the G2/M phase. The suppression of p-Akt accelerated the incidence of autophagy by downregulating mTOR, upregulating ULK1, and promoting autophagy. AMPK activation also inhibited the expression of mTOR while increasing that of ULK1, which promoted autophagy in cells. Meanwhile, the inhibition of Akt, and the activation of JNK and AMPK modulated the expression or the activation of Bcl-2 family members. Negative regulation of Bcl-2/ Mcl-1 and the reduction of the $\Delta \Psi_{m}$ initiated a program of mitophagy. These changes also engendered the activation of caspase-3/9, resulting in PARP cleavage and apoptosis. The activation of mitophagy was also involved in cell autophagy and apoptosis.

The current research had some limitations, including no in vivo experiments, only one cell line for experimental verification, and no non-cancer cell lines as negative controls. Future research needs to confirm the results of this study in more cell lines and in vivo research.
In conclusion, NCTD induces autophagy through the regulation of Bcl-2 family members, inducing mitochondrial dysfunction, inhibiting the PI3K/Akt/mTOR pathways, and activating the AMPK/ULK1 and JNK signaling pathways. The present study has evidenced cytotoxic effects of NCTD in A549 cells, and may pave the way for the use of NCTD as a novel treatment for NSCLC.

\section{Acknowledgments}

Funding: This work was supported by grants from the Medical Science Research Foundation of Guangdong Province in China (A2019535), the Administration of Traditional Chinese Medicine of Guangdong Province in China (20201078), the Science and Technology Planning Project of Guangzhou, China (202002030087), the Natural Science Foundation of Guangdong Province in China (2021A1515012521).

\section{Footnote}

Reporting Checklist: The authors have completed the MDAR reporting checklist. Available at http://dx.doi.org/10.21037/ atm-21-2360

Data Sharing Statement: Available at http://dx.doi. org/10.21037/atm-21-2360

Conflicts of Interest: All authors have completed the ICMJE uniform disclosure form (available at http://dx.doi. org/10.21037/atm-21-2360). The authors have no conflicts of interest to declare.

Ethical Statement: The authors are accountable for all aspects of the work in ensuring that questions related to the accuracy or integrity of any part of the work are appropriately investigated and resolved.

Open Access Statement: This is an Open Access article distributed in accordance with the Creative Commons Attribution-NonCommercial-NoDerivs 4.0 International License (CC BY-NC-ND 4.0), which permits the noncommercial replication and distribution of the article with the strict proviso that no changes or edits are made and the original work is properly cited (including links to both the formal publication through the relevant DOI and the license). See: https://creativecommons.org/licenses/by-nc$\mathrm{nd} / 4.0 \%$. 


\section{References}

1. Greenlee RT, Hill-Harmon MB, Murray T, et al. Cancer statistics, 2001. CA Cancer J Clin 2001;51:15-36.

2. Siegel R, Naishadham D, Jemal A. Cancer statistics, 2013. CA Cancer J Clin 2013;63:11-30.

3. Chen PL, Zhao T, Feng R, et al. Patterns and trends with cancer incidence and mortality rates reported by the China National Cancer Registry. Asian Pac J Cancer Prev 2014;15:6327-32.

4. Kadioglu O, Kermani NS, Kelter G, et al. Pharmacogenomics of cantharidin in tumor cells. Biochem Pharmacol 2014;87:399-409.

5. Liao HF, Su SL, Chen YJ, et al. Norcantharidin preferentially induces apoptosis in human leukemic Jurkat cells without affecting viability of normal blood mononuclear cells. Food Chem Toxicol 2007;45:1678-87.

6. Twig G, Elorza A, Molina AJ, et al. Fission and selective fusion govern mitochondrial segregation and elimination by autophagy. EMBO J 2008;27:433-46.

7. Kubli DA, Gustafsson AB. Mitochondria and mitophagy: the yin and yang of cell death control. Circ Res 2012;111:1208-21.

8. Hu ZY, Zhu XF, Zhong ZD, et al. ApoG2, a novel inhibitor of antiapoptotic Bcl-2 family proteins, induces apoptosis and suppresses tumor growth in nasopharyngeal carcinoma xenografts. Int J Cancer 2008;123:2418-29.

9. Liu X, Kim CN, Pohl J, et al. Purification and characterization of an interleukin-1beta-converting enzyme family protease that activates cysteine protease P32 (CPP32). J Biol Chem 1996;271:13371-6.

10. Chan NC, Salazar AM, Pham AH, et al. Broad activation of the ubiquitin-proteasome system by Parkin is critical for mitophagy. Hum Mol Genet 2011;20:1726-37.

11. Hanahan D, Weinberg RA. The hallmarks of cancer. Cell 2000;100:57-70.

12. Fan YZ, Zhao ZM, Fu JY, et al. Norcantharidin inhibits growth of human gallbladder carcinoma xenografted tumors in nude mice by inducing apoptosis and blocking the cell cycle in vivo. Hepatobiliary Pancreat Dis Int 2010;9:414-22.

13. Chen YN, Chen JC, Yin SC, et al. Effector mechanisms of norcantharidin-induced mitotic arrest and apoptosis in human hepatoma cells. Int J Cancer 2002;100:158-65.

14. Ye L, Yuan G, Xu F, et al. The small-molecule compound BM-1197 inhibits the antiapoptotic regulators Bcl-2/Bcl$\mathrm{xL}$ and triggers apoptotic cell death in human colorectal cancer cells. Tumour Biol 2015;36:3447-55.
15. Vera Y, Erkkila K, Wang C, et al. Involvement of p38 mitogen-activated protein kinase and inducible nitric oxide synthase in apoptotic signaling of murine and human male germ cells after hormone deprivation. Mol Endocrinol 2006;20:1597-609.

16. Edlich F, Banerjee S, Suzuki M, et al. Bcl-x(L) retrotranslocates Bax from the mitochondria into the cytosol. Cell 2011;145:104-16.

17. Rabinowitz JD, White E. Autophagy and metabolism. Science 2010;330:1344-8.

18. Tormo D, Checinska A, Alonso-Curbelo D, et al. Targeted activation of innate immunity for therapeutic induction of autophagy and apoptosis in melanoma cells. Cancer Cell 2009;16:103-14.

19. Melser S, Lavie J, Benard G. Mitochondrial degradation and energy metabolism. Biochim Biophys Acta 2015;1853:2812-21.

20. Beck JT, Ismail A, Tolomeo C. Targeting the phosphatidylinositol 3-kinase (PI3K)/AKT/mammalian target of rapamycin (mTOR) pathway: an emerging treatment strategy for squamous cell lung carcinoma. Cancer Treat Rev 2014;40:980-9.

21. Guo Y, Li Y, Shan Q, et al. Curcumin potentiates the antileukemia effects of imatinib by downregulation of the $\mathrm{AKT} / \mathrm{mTOR}$ pathway and BCR/ABL gene expression in $\mathrm{Ph}+$ acute lymphoblastic leukemia. Int J Biochem Cell Biol 2015;65:1-11.

22. Kumar D, Shankar S, Srivastava RK. Rottlerin-induced autophagy leads to the apoptosis in breast cancer stem cells: molecular mechanisms. Mol Cancer 2013;12:171.

23. Avalos Y, Canales J, Bravo-Sagua R, et al. Tumor suppression and promotion by autophagy. Biomed Res Int 2014;2014:603980.

24. Hahn-Windgassen A, Nogueira V, Chen CC, et al. Akt activates the mammalian target of rapamycin by regulating cellular ATP level and AMPK activity. J Biol Chem 2005;280:32081-9.

25. Copps KD, White MF. Regulation of insulin sensitivity by serine/threonine phosphorylation of insulin receptor substrate proteins IRS1 and IRS2. Diabetologia 2012;55:2565-82.

26. Shen B, He PJ, Shao CL. Norcantharidin induced DU145 cell apoptosis through ROS-mediated mitochondrial dysfunction and energy depletion. PLoS One 2013;8:e84610.

27. Zhao P, Dou Y, Chen L, et al. SC-III3, a novel scopoletin derivative, induces autophagy of human hepatoma HepG2 cells through AMPK/mTOR signaling pathway by acting 
on mitochondria. Fitoterapia 2015;104:31-40.

28. Seki E, Brenner DA, Karin M. A liver full of JNK: signaling in regulation of cell function and disease pathogenesis, and clinical approaches. Gastroenterology 2012;143:307-20.

Cite this article as: Liu Z, Li B, Cao M, Jiang J. Norcantharidin triggers apoptotic cell death in non-small cell lung cancer via a mitophagy-mediated autophagy pathway. Ann Transl Med 2021;9(12):971. doi: 10.21037/atm-21-2360
29. Bogoyevitch MA, Kobe B. Uses for JNK: the many and varied substrates of the c-Jun N-terminal kinases. Microbiol Mol Biol Rev 2006;70:1061-95.

(English Language Editor: J. Reynolds) 